\title{
Correction to: Laparoscopic-assisted ERCP following RYGB: a 12-year assessment of outcomes and learning curve at a high-volume pancreatobiliary center
}

\author{
Samer AIMasri ${ }^{1} \cdot$ Mazen S. Zenati $^{2} \cdot$ Georgios I. Papachristou $^{3} \cdot$ Adam Slivka $^{4} \cdot$ Michael Sanders $^{4}$. Jennifer Chennat ${ }^{4}$. \\ Mordechai Rabinowitz ${ }^{4}$. Asif Khalid ${ }^{4}$. Andres Gelrud ${ }^{5}$. John Nasr ${ }^{4}$. Savreet Sarkaria ${ }^{4} \cdot$ Rohit Das $^{4} \cdot$ Kenneth K. Lee $^{1}$.

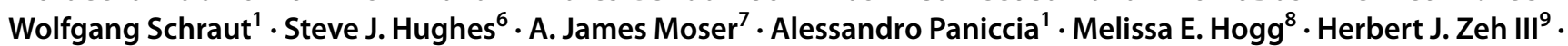 \\ Amer H. Zureikat ${ }^{1}$
}

Published online: 17 February 2021

@ The Author(s), under exclusive licence to Springer Science+Business Media, LLC part of Springer Nature 2021

\section{Correction to: Surgical Endoscopy https://doi.org/10.1007/s00464-021-08328-x}

This article was updated to correct a typo (131 patients not 31 patients) in the first sentence of the Results section of the abstract.
Publisher's Note Springer Nature remains neutral with regard to jurisdictional claims in published maps and institutional affiliations.

The original article can be found online at https://doi.org/10.1007/ s00464-021-08328-x.

Amer H. Zureikat

zureikatah@upmc.edu

1 Department of Surgery, University of Pittsburgh Medical Center, 5150 Center Ave., Suite 421, Pittsburgh, PA 15232, USA

2 Department of Surgery and Epidemiology, University of Pittsburgh, Pittsburgh, PA, USA

3 Department of Surgery, The Ohio State University, Wexner Medical Center, Columbus, OH, USA

4 Department of Internal Medicine, University of Pittsburgh, Pittsburgh, PA, USA
5 Department of Internal Medicine, Miami Cancer Institute, Gastro Health, Miami, FL, USA

6 Department of Surgery, University of Florida, Gainesville, FL, USA

7 Department of Surgery, Beth Israel Deaconess Medical Center, Boston, MA, USA

8 Department of Surgery, NorthShore Hospital System, Chicago, IL, USA

9 Department of Surgery, University of Texas Southwestern, Dallas, TX, USA 